\title{
Alteraciones en la fagocitosis de los leucocitos creviculares de pacientes diabéticos con enfermedad periodontal
}

Alteration in the phagocyte activity of crevicular leucocytes regarding to diabetic patients with periodontal disease

\begin{abstract}
Resumen
El objetivo de la presente investigación fue determinar la existencia de alteraciones en la fagocitosis de los leucocitos creviculares de pacientes diabéticos con Enfermedad Periodontal. Para tal efecto, se seleccionaron 56 individuos con Enfermedad Periodontal: 42 pacientes con diagnóstico de Diabetes Mellitus tipo 2 y 14 sin ella (grupo control), comprendidos entre 35 y 74 años de edad. Para la realización del estudio, se tomó como unidad de análisis los leucocitos provenientes del fluido crevicular de los pacientes en mención. Mediante lavados secuenciales; las muestras fueron analizadas microbiológica e inmunológicamente, teniendo como indicador, de la actividad fagocítica, a la Candida albicans ATCC. Hallándose que sólo el 22,02 $( \pm 7,62)$ de los leucocitos creviculares de diabéticos realiza una adecuada actividad fagocítica frente al 78,64 $( \pm 9,20)$ del grupo control. Asimismo el 78,21 $( \pm 7,84)$ de los leucocitos creviculares de diabéticos realiza la adherencia leucocitaria sin ingesta del patógeno frente a un $21,36( \pm 9,20)$ del grupo control. Resultando diferencias estadísticamente significativas en la adecuada fagocitosis del grupo de leucocitos creviculares de los diabéticos en relación al grupo control con un valor $\mathrm{t}=15,5(\mathrm{p}<=0,001)$, del mismo modo las diferencias estadísticas fueron significativas en relación de la adherencia leucocitaria sin ingesta del patógeno con un valor $t=15,4$ $(\mathrm{p}<=0,001)$ referente a los leucocitos creviculares de los pacientes diabéticos frente al grupo control.
\end{abstract}

\begin{abstract}
The goal of this investigation was to find out the alterations in the phagocyte activity of crevicular leucocytes regarding to diabetic patients with periodontal disease. For that reason, 56 patients with periodontal disease were selected: 42 of them with a diagnosis of Diabetes Mellitus - Type 2, and 14 patients without it (control group), from 35 to 74 years old. To performed the study, crevicular fluid of the mentioned patients was analyzed. By means of sequential washings the samples were analyzed microbiological and inmunologically, taking Candida albicans ATCC as indicator of the phagocyte activity. It was found that only a mean of $22.02( \pm 7.62)$ of diabetics crevicular leucocytes group have an adequate phagocyte activity compared to $78.64( \pm 9.20)$ of control group. In the same manner a mean of $78.21( \pm 7.84)$ of diabetics crevicular leucocytes group made adherence without ingestion of C.a. compared to only $21.36( \pm 9.20)$ of the control group. The differences between appropriated phagocytosis and ingestion activity of C.a. diabetics' crevicular leukocytes group and phagocytes of control group were statistical significant, $\mathrm{t}=15.5$ ( $\mathrm{c}<=0.001$ ), in the same manner the differences between leukocyte adherence without ingestion of C.a. of diabetic group and the control group, were statistical significant, $\mathrm{t}=15.4(\mathrm{p}<=0.001)$
\end{abstract}

\section{Introducción}

Las manifestaciones bucales, son frecuentes en diversos tipos de trastornos sistémicos, siendo el periodonto el más afectado, desencadenando en una enfermedad periodontal, las cuales presentan etapas de recesión y exacerbación ${ }^{(9-17)}$ que tiende a ser de acuerdo a la capacidad defensiva del huésped, función que esta representada por la acción que realizan los leucocitos creviculares, como primera barrera protectora ${ }^{(6-11)}$. Las pruebas evidencian que los leucocitos son células protectoras claves, contra la infección y/o inflamación ${ }^{(1-2-20)}$ por tal

\section{C.D. Jorge R. Colchado Carhuavilca'}

Departamento Académico Medico quirúrgico

Facultad Odontología. UNMSM. Lima Perú.

Correspondencia

C.D. Jorge Raúl Colchado Carhuavilca Facultad de Odontología UNMSM.

Av. Germán Amézaga s/n Lima 1 Perú. Telef. 511-2618993

E-mail: jcolchadoc@unmsm.edu.pe

Palabras Clave: Diabetes, crevice fluid, fagocitosis, leukocytes, adherence, periodontal disease.

Key words: Diabetes, crevice fluid, fagocitosis, leukocytic adherence, periodontal disease. 
creviculares, células críticas en la etiología de la enfermedad periodontal, es necesario conocer más sobre su función fagocítica, principal función protectora a nivel celular y tisular ${ }^{(26-27-29)}$, más aún al considerar a la Diabetes Mellitus tipo 2 como factor de riesgo en dicha patología ${ }^{(7-}$ 10-13-18-23), en consecuencia, es necesario identificar la existencia de alteraciones en alguno de los pasos de la fagocitosis y así proporcionar información científica que pueda contribuir a un adecuado tratamiento integral del paciente diabético, previniendo de esta forma el desarrollo de enfermedades periodontales.

\section{Materiales y Método}

Selección de Pacientes: La investigación fue llevado a cabo en 56 pacientes con Enfermedad Periodontal: 42 eran diabéticos tipo 2 y 14 eran no diabéticos (grupo control), comprendidos entre 35 y 74 años de edad. Se excluyó a todo paciente diabético con tratamiento periodontal previo así como también a aquellos que presentan movilidad dental a causa de trauma oclusal, a los portadores de procesos inflamatorios, que por si mismos o por tratamiento de otra entidad sistémica se encontrarán ingiriendo sustancias anti-inflamatorias que puedan alterar la composición del fluido crevicular. Ningún paciente recibió terapia antibiótica en los seis meses previos a la recolección de la muestra.

Recolección del Fluido crevicular: Se realizó 50 lavados secuenciales del crevículo gingival, con el medio de transporte celular (Suero Fetal de Ternera 20\%, M199) usando una pipeta convencional graduada en 10 ul (Finnpipette-LabSystem); y coleccionados en tubos Axigen, estériles. Luego, fueron centrifugados a 1000 rpm por 10 minutos y lavados 2 veces con PBS, se realizó el conteo celular en cámara de Neubauer con exclusión en Azul de Toluidina al 0,5\% como indicador de viabilidad celular, las células fueron concentradas en $10^{6}$ leucocitos/ml.

Actividad Fagocítica: La Candida albicans (C.a.) ATCC fue usada como partícula indicadora para determinar el número de fagocitos que contienen y se adhieren a leucocitos. La C.a. ATCC fue cultivada en agar Sabouraud, lavada y suspendida en PBS. Fue llevado a $90^{\circ} \mathrm{C}$ por 30 minutos sin destruir su apariencia general, nuevamente lavadas y suspendidas con PBS mas Suero Fetal de Ternera 20\% (SFT) a una concentración final de $5 \times 10^{8}$ C.a. / ml. A continuación, se tomó 0,2 ul de C.a. y se mezclo con 0,2 ul de suero AB Humano a $37^{\circ} \mathrm{C}$ por 30 minutos (opsonización). Se centrifugó y se lavó con PBS y fueron suspendidas con SFT. Luego, se tomó 10 ul de C.a. y se agrego a 10ul leucocitos a concentración de $10^{(8)}$ células $/ \mathrm{ml}$, se homogenizó y se colocó en láminas escavadas ( 3 pozos) y en una cámara húmeda, sin condiciones de agitación, a $37^{\circ} \mathrm{C}$ por 30 minutos (Fotos 1 y 2). Se retiró el sobre-nadante del $2^{\circ}$ y $3^{\circ}$ pozo, cubriéndose con $50 u l$ de solución Eosina Y 0,2\% + Azul Toluidina $0,4 \%$ observándose al microscopio a 100x. Se discriminó las C.a. adheridas (púrpuras) de las ingeridas (no coloreadas) (Fotos 3 y 4). El porcentaje fue calculado en 100 células viables.

\section{Resultados}

La glicemia de los pacientes diabéticos se encontraba compensada en un $78,6 \%$ y solo un $21,4 \%$ con valores clínicamente aceptables (Cuadro 1 y 2) La viabilidad celular de los leucocitos creviculares de los diabéticos fue de $88,48(+-3,76)$ y en los no diabéticos de $88,50(+-3,29)$.

En lo concerniente al total de leucocitos creviculares que realizan la fagocitosis del patógeno (Candida albicans), se puede apreciar que en los diabéticos la actividad fagocitaria fue mínima con $22,02(+-7,62)$ frente al mayor número de leucocitos activamente fagocitarios correspondiente a los no diabéticos 78,64(+- 9,20) (Cuadro 3 y 3.1).siendo la media y la desviación estándar significativamente diferentes,, además de contar con un $\mathrm{t}=15,5$ y un $\mathrm{GL}=54$ $y$ un $\mathrm{p}<=0,001$

Asimismo, la adherencia leucocitaria no fue complementada con la ingesta normal (fagocitosis) de la Candida albicans en los leucocitos creviculares de diabéticos con un 21,36 (+-9,20) a diferencia del grupo control (no diabéticos) en donde dicha adherencia fue complementada normalmente por la ingesta de la C.a. con un 78,21 $(+-7,84)$ (Cuadro 4 y 4.1) observándose diferencias significativas presentando un $\mathrm{t}=15.4$ y un $\mathrm{GL}=54$ y un $\mathrm{p}<=0,001$. Por último, se aprecia una mayor incidencia de fagocitosis en la gingivitis en comparación con la periodontitis tanto en diabéticos como en el grupo control. (Cuadro 5) Asimismo, existe una mayor adherencia leucocitaria sin ingesta del patógeno en la periodontitis del grupo de diabético y control en comparación con el proceso de la gingivitis (Cuadro 6)

\section{Cuadro 1. Distribución de pacientes según glicemia}

\begin{tabular}{cccc}
\hline $\begin{array}{c}\text { Glicemia } \\
(\mathrm{mg} / \mathrm{dl})\end{array}$ & frecuencia & $\begin{array}{c}\text { porcentaje } \\
\text { válido }\end{array}$ & $\begin{array}{c}\text { porcentaje } \\
\text { acumulado }\end{array}$ \\
\hline $80-95$ & 07 & 16,7 & 16,7 \\
$96-110$ & 12 & 28,6 & 45,2 \\
$111-125$ & 05 & 11,9 & 57,1 \\
$126-140$ & 09 & 21,4 & 78,6 \\
$141-155$ & 09 & 21,4 & 100,0 \\
TOTAL & 42 & 100,0 & \\
\hline
\end{tabular}

Cuadro 2. Presencia de micosis en los pacientes diabéticos

\begin{tabular}{lccc}
\hline \multicolumn{1}{c}{ Micosis } & frecuencia & $\begin{array}{c}\text { porcentaje } \\
\text { válido }\end{array}$ & $\begin{array}{c}\text { porcentaje } \\
\text { acumulado }\end{array}$ \\
\hline Presenta & 36 & 85,7 & 85,7 \\
No presenta & 06 & 14,3 & 100,0 \\
TOTAL & 42 & 100,0 & \\
\hline
\end{tabular}


Cuadro 3. Promedios de LFC según número de Candida albicans que fagocitan

\begin{tabular}{cccccc}
\hline Grupo & 1 c.a. & 2 c.a. & 3 c.a. & 4 c.a. & Total c.a. \\
\hline \multirow{2}{*}{ Diabéticos } & 11,74 & 06,95 & 02,98 & 0,38 & 22,02 \\
& $( \pm 4,60)$ & $( \pm 3,93)$ & $( \pm 2,05)$ & $( \pm 0,96)$ & $( \pm 7,63)$ \\
\multirow{2}{*}{ Control } & 36,69 & 26,23 & 12,00 & 1,69 & 78,64 \\
& $( \pm 6,49)$ & $( \pm 4,00)$ & $( \pm 3,16)$ & $( \pm 2,75)$ & $( \pm 9,20)$ \\
\hline
\end{tabular}

Cuadro 3.1. Valores descriptivos del grupo de LFC que realizan fagocitosis de C.a.

\begin{tabular}{lcccccc}
\hline \multicolumn{1}{c}{ Grupo } & media & $\begin{array}{c}\text { error } \\
\text { estándar }\end{array}$ & moda & $\begin{array}{c}\text { desviación } \\
\text { estándar }\end{array}$ & mínimo & máximo \\
\hline Diabéticos & 22,02 & 1,08 & 20 & 7,62 & 10 & 42 \\
Control & 78,64 & 2,46 & 84 & 9,20 & 63 & 90 \\
\hline
\end{tabular}

Cuadro 4. Promedios de LFC que realizan adherencia sin ingesta de C.a.

\begin{tabular}{cccccc}
\hline Grupo & 1 c.a. & 2 c.a. & 3 c.a. & 4 c.a. & Total c.a. \\
\hline \multirow{2}{*}{ Diabéticos } & 13,10 & 33,50 & 20,95 & 8,01 & 78,21 \\
& $( \pm 9,27)$ & $( \pm 9,50)$ & $( \pm 7,83)$ & $( \pm 6,53)$ & $( \pm 7,84)$ \\
\multirow{2}{*}{ Control } & 08,38 & 08,08 & 02,69 & 0,92 & 21,36 \\
& $( \pm 2,87)$ & $( \pm 5,85)$ & $( \pm 1,65)$ & $( \pm 1,55)$ & $( \pm 9,20)$ \\
\hline
\end{tabular}

Cuadro 4.1. Valores descriptivos del grupo de LFC que realizan adherencia sin ingesta de C.a.

\begin{tabular}{lcccccc}
\hline Grupo & media & $\begin{array}{c}\text { error } \\
\text { estándar }\end{array}$ & moda & $\begin{array}{c}\text { desviación } \\
\text { estándar }\end{array}$ & mínimo & máximo \\
\hline Diabéticos & 78,21 & 1,21 & 80 & 7,84 & 58 & 90 \\
Control & 21,36 & 2,46 & 16 & 9,20 & 10 & 37 \\
\hline
\end{tabular}

Cuadro 5. Promedios de LFC que fagocitan C.a. según grupo y condición periodontal

\begin{tabular}{clcccc}
\hline Grupo & Condición & 1 c.a. & 2 c.a. & 3 c.a. & 4 c.a. \\
\hline \multirow{2}{*}{ Diabéticos } & Gingivitis & 14,2 & 17,5 & 4,5 & 04 \\
& Periodontitis & 11 & 08 & 04 & 04 \\
\multirow{2}{*}{ Control } & Gingivitis & 40 & 34 & 13 & 04 \\
& Periodontitis & 36,57 & 28 & 11 & 04 \\
\hline
\end{tabular}

Cuadro 6. Promedios de LFC que realizan adherencia sin ingesta de C.a. según grupo y condición periodontal

\begin{tabular}{clcccc}
\hline \multicolumn{1}{c}{ Grupo } & Condición & 1 c.a. & 2 c.a. & 3 c.a. & 4 c.a. \\
\hline \multirow{2}{*}{ Diabéticos } & Gingivitis & 11,53 & 34,6 & 23,4 & 13 \\
& Periodontitis & 15,76 & 34,6 & 21,2 & 9,92 \\
\multirow{2}{*}{ Control } & Gingivitis & 07 & 7,5 & 04 & 04 \\
& Periodontitis & 10 & 09 & 04 & 04 \\
\hline
\end{tabular}

16 Odontol. Sanmarquina 2008; 11(1): 14-18

\section{Discusión}

Este estudio permitió confirmar la presencia de un gran número de células obtenidas de los lavados creviculares, tanto en los pacientes diabéticos como en los controles, siendo la mayoría de las células de defensa neutrófilos PMNs, tal y conforme se reportaron en otros estudios ${ }^{(25)}$. Todo paciente diabético mantenía valores de glicemia aceptables tanto por la OMS y la $\mathrm{ADA}^{(3-4-5)}$. La investigación tuvo como indicador de la fagocitosis a la Candida albicans, teniendo en cuenta que, la Diabetes Mellitus es un factor predisponente a Candidiasis oral ${ }^{(8-13)}$ (Cuadro 2) hallándose, que el sistema inmunológico de los pacientes diabéticos no realiza una adecuada ingestión de la Candida albicans probablemente por presentar problemas en el sistema inmunológico del diabético y no reconoce al patógeno como antígeno, incrementando la posición de que existe una alteración en la fagocitosis en los leucocitos creviculares que pueden condicionar a una Enfermedad Periodontal. La pobre ingesta de los patógenos por parte de los leucocitos creviculares de diabéticos en comparación con el grupo control hallados en el presente estudio, nos demuestra que existen alteraciones fagocitarias (Cuadro 3 y 3.1). Este hallazgo se respalda en la mayor adherencia sin ingesta de patógenos por parte de los leucocitos de los mismos diabéticos en comparación con los de control (Cuadro 4 y $4.1)$; probablemente debido a diversos factores como la microangiopatía diabética $^{(24)}$ hiperglicemia, factores moleculares - inmunológicos como el TNF alfa $^{(16)}$ que impide una respuesta tisular efectiva ante factores irritativos, una menor afluencia de células de defensa y una irregular captación de glucosa extracelular presente en el fluído crevicular ${ }^{(12-}$ 21-22-28), fuente principal de energía para los fenómenos de glucólisis, y que impedirían una adecuada realización de la Quimiotaxis 1o cual desencadenaría en una probable Fagocitosis frustrada ${ }^{(12-19-25-27)}$ en el diabético en donde el fagocito 
se adhiere al patógeno y degranula, descargando su contenido intracelular de lisosomas y gránulos específicos y azurófilos, pero no fagocita ${ }^{(6)}$. Por último, en lo que concierne a la fagocitosis en relación a los diferentes tipos de enfermedades periodontales (Cuadro 5y 6), se evidencia una mayor actividad fagocítica en la gingivitis en comparación con la periodontitis y por otro lado la existencia de una mayor adherencia leucocitaria sin ingesta de patógenos en la periodontitis en comparación con la gingivitis, tanto en los diabéticos como en el control, esto puede ser considerado por su diferencia etiológica y/o tiempo del proceso patológico.
Al término del estudio se llegó a las siguientes conclusiones:

. La Diabetes Mellitus tipo 2 provoca una reducción en la función fagocítica de los leucocitos creviculares condicionando a una enfermedad periodontal.

. La adherencia leucocitaria, sin complemento fagocitario (ingesta del patógeno), son evidencias claras de que existen alteraciones en la fagocitosis de los leucocitos creviculares de los pacientes diabéticos con enfermedad periodontal.

. En la Gingivitis, se produce una mayor acción fagocítica que en la pe- riodontitis, tanto en el diabético como en los no diabéticos.

. En la periodontitis, se produce una mayor adherencia leucocitaria sin ingesta del patógeno en comparación con la gingivitis, tanto en diabéticos como en no diabéticos.

- La incidencia y la gravedad de la enfermedad periodontal está condicionada a numerosos factores, pero en la Diabetes Mellitus tipo 2 lo más preocupante es la deficiencia inmunológica que existe ante la agresión de microorganismos patógenos, debido a una deficiente fagocitosis por parte de los leucocitos creviculares.

\section{Alteraciones en la fagocitosis de los leucocitos creviculares de pacientes diabéticos con enfermedad periodontal}

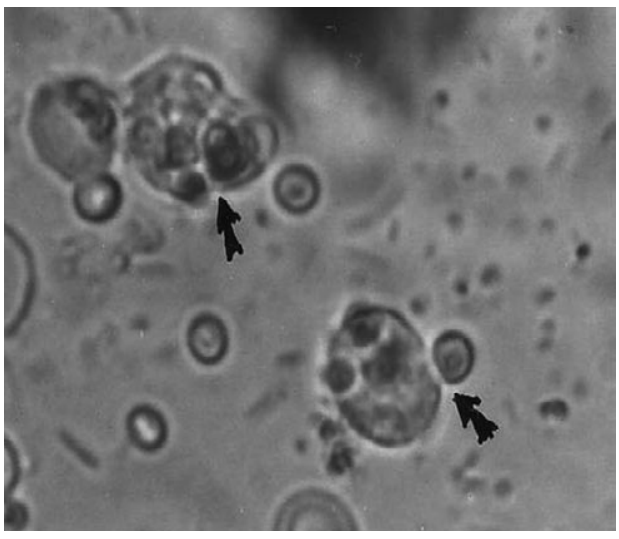

Foto 1: Leucocito del Fluido Crevicular (LFC) en plena fagocitosis: Iniciando englobamiento de la C. albicans (abajo) y concluyendola (arriba). Magnificación $1000 x$

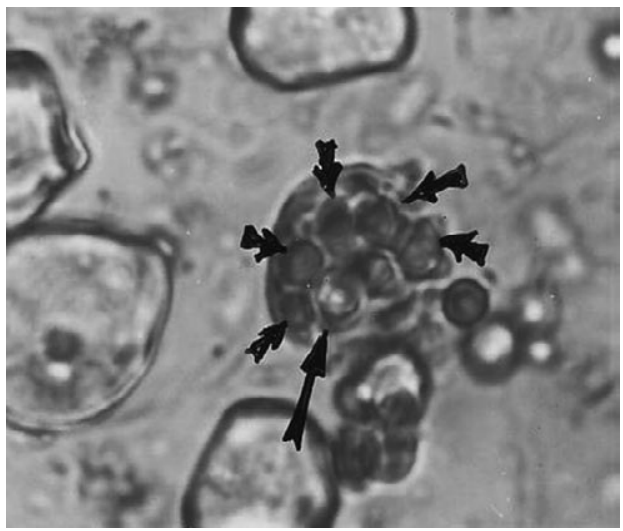

Foto 3: LFC se evidencia las C. albicans adheridas no fagocitadas (color púrpura - flecha corta) de las fagocitadas (no coloreadas - flecha larga) Coloración Eosina Y + Azul toluidina $(1000 x)$

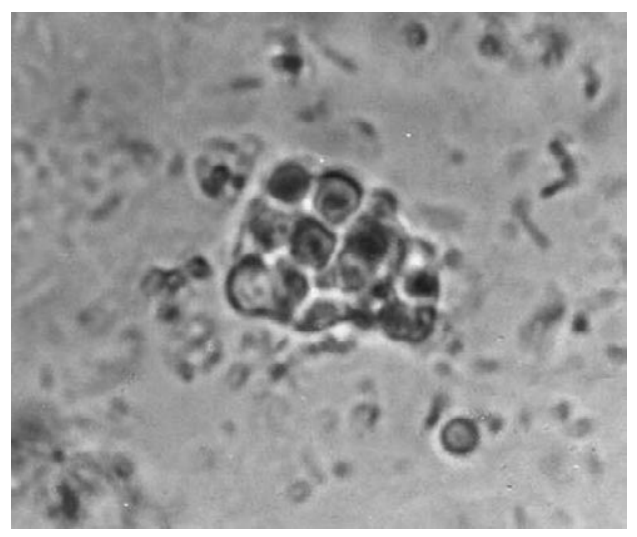

Foto 2: LFC culminando la fagocitosis. Magnificación $1000 x$

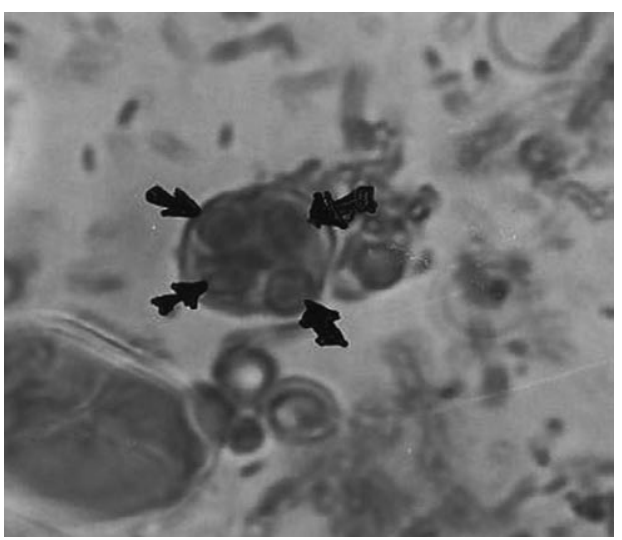

Foto 4: LFC de diabético se evidencia que sólo las C.albicans son adheridas (color púrpura - flechas) y no son fagocitadas. Magnificación 1000 x 


\section{Referencias Bibliográficas}

1. Aguilar Serrano Pérez Estrada "Alteraciones de la Fagocitosis en el Asma Bronquial “ Rev. Neumología Y Cirugía de Tórax Vol 56(3):59 - 64. 1991.

2. Alzamora L. Escajadillo C. "Manual de Prácticas - Curso Inmunoquímica E Inmunología Y Geología “ $9^{a}$ Ed. UNMSM Lima - Perú 1999

3. American Diabetes Association. Diagnosis and classification of diabetes mellitus. Diabetes Care. 2005: 29 (Suppl. 1): $37-s 42$.

4. American Diabetes Association Expert Committee on the Diagnosis and Classification of Diabetes Mellitus. Committee Report. Diabetes Care 1997: 20: 1183-1197.

5. American Diabetes Association Expert Committee on the Diagnosis and Classification of Diabetes Mellitus. Follow up Report on the Diagnosis of Diabetes Mellitus. Diabetes Care 2003 : 26: 3160-3167.

6. Barrios Gustavo. Odontología: Su Fundamento Biológico. Edit. Iatros Colombia 1991

7. Barroso I. Genetics of type 2 diabetes. Diabet Med 2005: 22: 517-535.

8. Belazi Maria et al. Candidal overgrowth in diabetic patients: potential predisposing factors Mycoses. 2005; Vol. 48(3): 192-196

9. Carranza F. Periodontología Clínica de Glickman" 6 a ed. México. Interamericana 1998

10. Cerda J. Vásquez de La Torre - Malacara "La enfermedad periodontal en la diabetes mellitus no insulino dependiente - el efecto de la edad y el tiempo desde el diagnóstico" J.Periodontol 1995; Vol. 65 (11):991 - 5

11. Cimasoni G. "Crevicular fluid undated" Monograf Oral Scienc. 1983 Vol.12(1)

12. Cohen - Leony - Simpson "Phagocytic cells in periodontal defense" J. Periodontol. Oct 1985. Vol 56(10): 612 -615 .

13. Dorocka-Bobkowska B. et al. Noninsulin-dependent diabetes mellitus as a risk factor for denture stomatitis
Journal of Oral Pathology \& Medicine Vol 25(8) Sep 1996: 411-415

14. Emrich-Shlossman-Genco “Periodontal disease in non-insulin- dependent diabetes mellitus" J.Periodontol. Feb.1991 Vol.62(2):123-131

15. Esmann V. "The polymorphonuclear leukocyte in diabetes mellitus"J. Clin Chem. Clin.Biochem. Sep 1983 Vol.21(9): 561-567

16. Fusanori Nishimura Et Al (2003) Periodontal disease and diabetes mellitus: the role of tumor necrosis factor-a in a 2-way relationship. Journal Of Periodontology January 2003. Vol. 74(1), 97-102

17. Genco-Goldman-Cohen Periodoncia. Mexico: Edit. Interamericana. 1993

18. Grossi-Genco “Periodontal disease and diabetes mellitus: a two-way relation ship" Ann. Periodontal July 1998 Vol.3(1):51-61

19.Higgs - Mc Call - Youlten "A chemotactic role for prostaglandins released from polymorphonuclear leukocytes during phagocytosis" Br. J. Pharmacol 1975; 53:539

20. Male David "Inmunology" 2a ed. Londres: Edit Sanda. 1991

21. Matarasso - Cafiero - Bizzani - Nicolo "The role of phagocytic cells in periodontal disease" Minerva Stomatol 1991; Vol.40(4): 203 - 210

22. Mc Mollen-Van Dylre-Genco "Neutrophil chemotaxis in individuals with advanced periodontal disease and a genetic predisposition to Diabetes Mellitus" J.Periodontol 19981; Vol.52(4):167 - 173

23. Nishimura-Takahashi "Periodontal disease as a complication of Diabetes Mellitus" Ann Periodont. July 1998 Vol $3(1): 20-29$

24. Romero Rivas - Gutiérrez Manay J.- Rojas "Diagnóstico precoz de la diabetes mediante estudio histológico de la gíngiva" Rev. Alma Mater UNMSM. Agosto 1994 Vol.8: 65 - 78

25. Sigush-Klinger-Holtz-Süss “In vitro phagocytosis by crevicular phagocytes In many forms of periodontitis" J.Periodontol. 1992 63:496 - 501
26. Tan - Anderson - Watanakunakorn "Neutrophil dysfunction in Diabetes Mellitus" J Lab. Clin. Med. Jan 1975 Vol.85(1):26-33

27. Van Dyke - Zinney - Winkel “Neutrophil function in localized Juvenil Periodontitis: phagocytosis, superoxide production and specific granule release" J.Periodontol. Nov.1986 903 - 908

28. Wierusz - Wysocka - Wysocki "Phagocytosis, bactericidal capacity and superoxide anion $\left(\mathrm{O}_{2}^{-}\right)$production by polymorphonuclear neutrophils from patients with Diabetes Mellitus" Folia Aematol. Int. May. Klin. Morphol. Blutforsch. 1985 Vol 112(5):658 - 668

29. Wilson-Reeves W.G. “Neutrophil phagocytosis and killing in insulindependent diabetes" Clin. Exp. Inmunol. Feb 1986 Vol 63(2): 478-484

30. Zachariasen R.D. “Diabetes Mellitus And Periodontal Disease" Compendium. May 1991 Vol.12(5):324, 326-328.

Recibido :07-07-2008

Aceptado para publicación: 13-08-2008 